\title{
Educación para el consumo sostenible desde la pedagogía crítica
}

\author{
Miguel Martín-Sánchez \\ https://orcid.org/0000-0002-9370-1732 \\ Laura Casares-Ávila \\ https://orcid.org/0000-0003-3362-2888 \\ Jorge Cáceres-Muñoz \\ http://orcid.org/0000-0002-2405-9731
}

\section{Resumen}

La educación para el consumo desde una perspectiva crítica es un tema serio que necesita ser estudiado y trabajado por todos los miembros de la sociedad educativa. Este trabajo plantea dos objetivos. Por un lado, realizar un análisis de la sociedad actual en clave de consumo. En la actual sociedad occidental el consumo de bienes se ha convertido en un factor de integración en la sociedad y se ha consagrado como la llave para acceder al mundo de primer orden. Las lógicas del mercado de consumo se han instaurado en la educación occidental creando una pedagogía consumista. Por otro lado, se pretende justificar la necesidad de una pedagogía crítica para el consumo como modelo pedagógico dirigido a alfabetizar económicamente a niños y jóvenes mediante el fomento del pensamiento crítico y el fortalecimiento del sistema educativo público para un adecuado y equitativo desarrollo individual y social. En cuanto al método, este trabajo se ha llevado a cabo desde una perspectiva y análisis crítico de las teorías educativas y sociales que abordan el consumo y sus consecuencias para la sociedad del siglo XXI. Como resultado se presenta el planteamiento de construir y defender críticamente la escuela y la educación a través de acciones coordinadas entre el alumnado y el profesorado que logre el empoderamiento para ser críticos, reflexivos y socialmente responsables. Se concluye que educar para el consumo responsable implica el compromiso de toda la comunidad educativa para lograr un cambio social.

\section{Palabras clave}

Consumismo - Educación para el consumo - Escuela - Pedagogía crítica.

1 - Universidad de Extremadura, Cáceres, España. Contactos: miguelmartin@unex.es; casaresavila@gmail.com; jorgecm@unex.es 


\section{Education for sustainable consumption from critical pedagogy}

\section{Abstract}

Education for consumption from a critical perspective is a serious issue that needs to be studied and worked on by all members of the educational society. This work raises two objectives. On the one hand, to carry out an analysis of the current society in terms of consumption. In today's Western society, the consumption of goods has become a factor of integration in society and has been established as the key to access the first-order world. The logic of the consumer's market has been established in Western education creating a consumer pedagogy. On the other hand, it is intended to justify the need for a critical pedagogy for consumption as a pedagogical model aimed at making children and young people economically literate by promoting critical thinking and strengthening the public educational system for an adequate and equitable individual and social development. Regarding the method, this work has been carried out from the perspective and critical analysis of educational and social theories that address consumption and its consequences for the 21 st century society. As a result, the approach of constructing and critically defending the school and education is presented through coordinated actions between students and teachers that achieve the empowerment to be critical, reflective and socially responsible. Conclusion is that educating for responsible consumption requires the commitment of the entire educational community in order to achieve social change.

\section{Keywords}

Consumerism - Education for consumption - School - Critical pedagogy.

\section{Introducción. Neoliberalismo y mercado de consumo}

Este trabajo tiene como primer objetivo ahondar en las estructuras en las que se basa la sociedad actual en clave de consumo. En paralelo, se pretende un segundo objetivo, desde el abordaje a distintos elementos políticos, económicos y sociales que describiremos en las próximas líneas; buscamos justificar una pedagogía crítica, potenciadora de una formación para el consumo, en contraposición a una pedagogía consumista. La pedagogía crítica está en condiciones de activar la posibilidad de armar una estrategia coherente de formación ciudadana con orientación a una equitativa y estable forma de ver el consumo. Por tanto, estas líneas no pretenden presentar recetas infalibles contra el desmedido sentimiento y acción consumista que inunda la sociedad occidental, sino ofrecer razones de la necesidad de un cambio de mentalidad a través de la pedagogía. La solución no se encuentra exclusivamente en la escuela, sino en múltiples escenarios y desde diferentes 
acciones: el conocimiento de la realidad social actual y de las dinámicas del mercado, los cambios curriculares y prácticas metodológicas innovadoras colocando al objetoenseñado como objeto-problema que hay que solucionar para hacer crecer el componente crítico y actitudinal en los estudiantes, las reformas en la formación del profesorado, el trabajo colaborativo con plataformas sociales y cívicas, la educación familiar, o los medios de comunicación y el análisis de su influencia.

Hace tiempo que Susan George (2003) advirtió que la sociedad se hallaba enclavada en un sistema que no consigue dar respuestas a las necesidades reales, puesto que se trata de un proceso de pseudolibertad caracterizado por transacciones transfronterizas de capitales y de bienes y servicios, donde se obedece únicamente a la ley de la oferta y la demanda y en el que todo se compra y se vende. El neoliberalismo ha desarrollado unas lógicas mercantiles sustentadas en la acumulación de bienes y en el capital. En este consumo acelerado, la globalización neoliberal ha ejercido una poderosa influencia en muchas de las políticas educativas noroccidentales, lo que ha provocado un cambio hacia una economía del conocimiento, una transformación capitalista del conocimiento en el que se compra y se vende la creación, la adquisición, la innovación y la transmisión del conocimiento (OLSSEN; PETERS, 2005), rápido, calculado, eficiente y rentable (BESLEY; PETERS, 2005). Así, aprendemos para trabajar y el aprendizaje es un bien de consumo, cobrado, donde se plantean nuevas formas de esclavitud, donde se confunde el entusiasmo con la estafa, el oficio con la mendicidad, donde se sacrifican seres humanos al mercado a cambio de una ración de subsistencia, de una promesa de futuro, donde tener trabajo y cualquier sueldo se presenta como una bendición. Se presenta la falsa promesa de trabajar a cualquier precio para consumir, de tal manera que se aumenta la deuda, y con ella el sometimiento al sistema. La trivialidad y la mediocridad hacen posible que las lógicas neoliberales del mercado laboral puedan llevar a cabo atrocidades de todo tipo. Y así, el poder del consumo se inocula en la sociedad.

El poder neoliberal en relación con los hábitos de consumo no solo representa una importante máquina productora de bienes de consumo, sino también una constructora de consumidores, una educadora aplicada. La compañía norteamericana Disney es un buen ejemplo de la construcción de consumidores a través de procesos pedagógicos modificados en los que enseñan formas específicas de ser y comprar (SANDLIN; GARLEN, 2017), y que ejerce su influencia en los niños y adultos (MAUDLIN; SANDLIN; THALLER, 2012) convirtiéndoles en consumidores pasivos de su propaganda política y su forma de entender la cultura con valores como familia, género, etnicidad, entrenamiento y consumo, y que no permanece ajena al poder, la ideología y la política (GIROUX; POLLOCK, 2010), sino que presenta un ideal de sociedad que reduce al sujeto a un consumidor homogéneo sin actitud crítica ni herramientas necesarias para cuestionarse otras formas de ser y entender el mundo y la cultura.

El consumo de bienes no sólo se ha convertido en un factor de integración en la sociedad más próxima del individuo, sino que se ha consagrado como la llave para acceder al mundo de primer orden, es decir, a la parte privilegiada de la globalización (GEORGE, 2003). 
La sociedad occidental presenta y ensalza un sujeto caracterizado por el individualismo y el materialismo, donde la globalización y el capitalismo han colocado al mercado y los intereses económicos por encima de las personas. Los medios de comunicación transmiten una pedagogía consumista basada en un modo de vida sensorial, hedonista y egoísta sustentado en sueños de progreso que fomentan formas superficiales de vivir sostenidas en la exaltación de las formas frívolas del consumo. El neoliberalismo y su abanderado consumismo ejercen una pedagogía consumista que atenaza al individuo y lo convierte en un ser débil y desprotegido a merced de las tendencias del mercado, que le deslumbra con sueños atractivos de grandeza y bienestar social y personal.

El significante de los sueños de las nuevas generaciones ya no son los ideales de honradez, honestidad e igualdad, sino que se han ido subyugando hacia el bien individual, el egoísmo, el propio beneficio, los códigos morales individuales, auspiciado bajo el paraguas del capitalismo y el consumismo, explotando al ser humano para conseguir un mayor beneficio económico, una falsa prosperidad y felicidad enmascarada en progreso y bienestar con la falsa ilusión de tener más, producir más y consumir más para ser feliz.

Los centros comerciales se crearon para ser máquinas de fantasía, donde todo debía estar fusionado, anulando las distancias y el tiempo, y generando una confusa colección de fragmentos y experiencias, dispuestos y organizados para ser consumidos y no vivenciados ni representados (CRAWFORD, 1992).

A todo ello se sumó la instauración de la publicidad y el marketing como instrumentos de información y persuasión, convirtiendo los productos y bienes en auténticos objetos de deseo, invitando e incluso incitando a la población a consumir necesidades externamente construidas. La publicidad, la televisión, los medios de comunicación no son inocentes e inocuos, sino que se convierten en agentes educativos informales que "[...] promote and reinforce the hegemonic belief that the consumption of goods and services is the primary means of fulfilment and happiness" ${ }^{2}$ (WRIGHT; SANDLIN, 2017, p. 83) e impulsan la necesidad de aumentar la producción y el consumo.

Se consume para ser feliz, o al menos esa es la idea que el capitalismo propaga. Sin embargo, a pesar de que existe cierta relación entre el bienestar psicológico y la riqueza material, Górnik-Durose (2006) indica que la riqueza cumple con las necesidades humanas en relación con el confort, aunque matiza que cuando los sujetos consiguen un cierto nivel de riqueza, no aumenta la felicidad obteniendo más riquezas, puesto que el excedente obtenido de las posesiones obliga a los usuarios a luchar con los costos psicológicos adicionales, siendo necesaria una educación que supere estas contradicciones.

El ser humano ha llegado a transformarse en un bien de consumo, enajenado, una nueva especie, denominada Homo Consumiens, el consumidor final, cuyo objetivo es acaparar más, gastar más, usar más, tener más y compensar su vacío, ansiedad y soledad (FROMM, 1981). Y en esta vorágine consumista, los niños son especialmente vulnerables, al carecer de las herramientas necesarias para el filtrado de los productos publicitarios. En este sentido, Calvert (2008) indica la necesidad de alfabetizar críticamente a los niños en

2- "[...] promueven y refuerzan la hegemónica creencia de que el consumo de bienes y servicios es el medio para alcanzar la felicidad y sentirse realizado" (traducido por los autores). 
relación con la publicidad y el consumo, especialmente por su influencia en la toma de decisiones a la hora de comprar y consumir.

El consumo construye la forma en la que se ve el mundo (CRAWFORD, 1992), por ello es necesario otorgar a las nuevas generaciones herramientas críticas para la vida, que les permitan articular las complejidades políticas, económicas, sociales, culturales que se van a ir encontrando, permitiéndoles alcanzar cierta claridad sobre quiénes son y cómo se representan en el entorno que les envuelve. Para ello, la reflexión política, la participación ciudadana y la educación de las personas como consumidores, deben convertirse en piezas clave de la construcción de identidades (ALONSO; FERNÁNDEZ, 2010).

Frente al dominio del discurso, las prácticas y las políticas educativas por parte de las élites de poder, que colocan al mercado y al beneficio por encima de otras consideraciones y que entienden el aprendizaje en términos de crecimiento económico (DAMIANIDOU; PHTIAKA, 2016; HURSH; HENDERSON, 2011), salimos en defensa de una pedagogía crítica que proteja, defienda y forme a la ciudadanía, para tomar decisiones libres, fundamentadas en información veraz y acompañada de racionalidad. Desde esta forma de entender la pedagogía, puede hablarse de un nuevo modelo de gestión del consumo y, con ello, de las necesidades de los consumidores y de los recursos del planeta.

\section{Postmodernidad y consumo, o cómo se compra y se vende la educación}

Featherstone (1988) ya anunció que la aparición de la postmodernidad significaba, además de la ruptura con la modernidad, el surgimiento de una nueva estructura social con nuevos principios organizativos, entre ellos el consumo. Dentro del compendio postmodernista resultó de elevada relevancia la instauración del concepto de Nueva Economía (PORCELLI; MARTÍNEZ, 2015), que acuñó el economista Brian Arthur y popularizó Kevin Kelly, fundador y director ejecutivo de la revista estadounidense Wired.

Alvin Toffler (1970) vaticinó que estaba llegando la civilización de usar y tirar. Los productos, las necesidades, las vivencias se crean actualmente para ser usados una sola vez o durante un breve periodo de tiempo. Lo efímero, como advirtió Lipovetsky (2002), es el máximo exponente de la postmodernidad.

El mercado de consumo, además de generar exceso y despilfarro, fomenta el desarrollo de emociones positivas vinculadas a la compra compulsiva y conduce al desprendimiento de aquellas adquisiciones que dejan de ser atractivas, para poder adquirir otras nuevas y más estimulantes (BAUMAN, 2007), en una suerte de modernidad líquida en constante cambio que afecta a la forma de entender la educación y la sociedad (BAUMAN, 2005). Esta espiral sumerge a la población en una sociedad de consumo y como consecuencia, en una sociedad de endeudamiento, puesto que el individuo llega a gastar más allá de sus recursos monetarios para poder participar de este ciclo y poder acceder a productos, bienes y servicios que considera propios del grupo social al que pertenece o desea pertenecer.

El capitalismo mundial ha conocido diversas crisis financieras a lo largo de la historia. Como causa de esta última se puede señalar el crecimiento desmedido de la llamada burbuja financiera, originada principalmente por el vertiginoso avance de las 
tecnologías de la información y la comunicación (BEINSTEIN, 2009), y el interés del mercado y las grandes empresas en utilizar la tecnología y los productos tecnológicos dentro de la escuela, generando dependencias entre lo que se vende y se compra, el hardware y el software que producen y comercializan determinadas empresas, creando un mercado tecnológico de producción al que se subyuga la escuela, implementando lógicas políticas de la tecnología en tanto que se emplean para desarrollar estructuras organizativas basadas en la jerarquía y la autoridad (SALTMAN, 2016) basado en el pensamiento neoliberal del mercado y el consumo. Y la escuela se doblega a las políticas educativas neoliberales, que parece que han venido para quedarse en el sistema educativo actual (ARRIAZU; SOLARI, 2015; BERNAL AGUDO; LACRUZ, 2012; JONES et al., 2008; MAISURIA, 2014; SANTA CRUZ; OLMEDO, 2012; VIÑA0, 2001, 2012).

Esta situación de declive económico encuentra su lógica en el capitalismo mismo, puesto que hacer del capital el motor de la economía y de su acumulación la parte esencial del desarrollo humano conduce a un inevitable desequilibrio (BERLANGA, 2010). Este desequilibrio no solo se representa a nivel monetario sino también a nivel energético, alimentario e incluso ecológico, enmascarado en falsas promesas de crecimiento económico sostenible basado en tecnofilia, eficiciencia y ecología para imponer los formatos electrónicos controlados por las grandes industrias en detrimento de otros soportes como el papel (SALTMAN, 2016).

En esta lucha por imponer una pedagogía consumista, el neoliberalismo ha instrumentalizado la publicidad para convertirla en una herramienta educativa, instaurando el denominado neuromarketing. Este concepto es entendido como un instrumento basado en anticipar las reacciones que las campañas publicitarias producen sobre el cerebro del espectador. Se trata de una técnica de investigación cerebral que busca analizar cómo un estímulo externo genera expectativas sobre un producto o servicio y cómo tales expectativas provocan el deseo de adquirirlo (LINDSTROM, 2010).

Un estudio llevado a cabo por Badaoui, Lebrun y Bouchet (2012), con una muestra de 1063 adolescentes franceses, confirmó que existe una influencia significativa de los medios de comunicación y la música sobre la identificación de los adolescentes con determinados grupos y, en consecuencia, con el consumo de determinadas marcas. Se observó que cuanto más fuertes eran las influencias mediáticas y musicales, más fuerte era la identificación del grupo de adolescentes con determinados productos y marcas.

La cuestión estriba en diferenciar lo necesario de lo inútil y la libertad de pensamiento de la alienación. Se puede hablar de ciudadanos-espectadores-deseadores u Homo Consumiens, cuyo estado natural es la insatisfacción constante (COHEN, 2014). La televisión, y especialmente la publicidad que mueve millones de dólares al año no se limita a vender productos, sino que moldea la imagen, la construcción de identidades, los valores, las comunidades y los grupos, construyendo un mensaje pedagógico que busca educar para consumir constantemente (GIROUX; POLLOCK, 2010). Es la creación perfecta del sujeto dependiente de los bienes de consumo, un sujeto educado desde la infancia en la dependencia del mercado, que le dice lo que tiene que comprar y lo que tiene que poseer para pertenecer a determinado grupo social, sin un ápice de pensamiento o actitud 
crítica ante la construcción de la identidad colectiva e individual que presenta la industria publicitaria.

El consumo, sobre todo en los grandes almacenes, se ha ido instaurando como una actividad de ocio y tiempo libre para muchos niños, dedicándole un mayor porcentaje de tiempo que a leer, jugar al aire libre, o asistir a reuniones infantiles (SCHOR, 2005). El consumismo es la ideología dominante inculcada a los menores y adultos representando el centro de la vida y la cultura global en el mundo desarrollado (SPRING, 2003).

\section{Pedagogía crítica y educación para el consumo}

El propio ser humano se ha convertido en producto de consumo, puesto que hay que ser el mejor con el mejor currículo para alcanzar el mejor precio en el mercado laboral y social. De manera correlacional, a estos cambios económicos se han ido produciendo transformaciones en los valores, que han obligado al individuo a adaptarse, incluirse e integrarse en este nuevo sistema (COHEN, 2014). La respuesta crítica a este cambio de valores impuesto por el mercado está ligada a la ontología del ser y a su formación ética.

Tomando como base la fundamentación teórica sobre los diferentes niveles del desarrollo económico (DENEGRI et al., 2006; WAINES, 1984), es posible articular una pedagogía crítica que establezca un modelo de intervención dirigido a alfabetizar a niños y jóvenes mediante diversas actividades adaptadas a su edad, aplicables a sus vidas cotidianas y basadas en el aprendizaje crítico, de tal manera que les permita adquirir conceptos básicos de economía, estrategias de razonamiento económico, habilidades como consumidores y actitudes proactivas. Así, estariamos desarrollando y orientando el sentido crítico de la educación, el crecimiento y madurez personal, que consigue un pensamiento y criterio propio, ante las prácticas deshumanizadoras del mercado y el capital, contra el intelectualismo y la moral.

Una Educación para el Consumo desde una perspectiva crítica permite plantear la acción de consumir en términos de aprendizaje y de mejora, y no como esclavos de la economía capitalista globalizada que camina hacia objetivos del trabajo y el consumismo, perdiendo la perspectiva, por ejemplo, de que las escuelas públicas no son lugares cuya existencia se fundamenta en la acumulación de ganancias (SALTMAN, 2016).

En una sociedad donde se presenta la necesidad de consumir para vivir, cuando no hay educación en valores de consumismo racional sino compulsivo, donde los niveles de consumo se convierten en algo patológico, se evidencia que las ansias de consumir tratan de ocultar un sentimiento de vacío generado por la constante presión de la sociedad por consumir (FROMM, 1986), justificando, una vez más, la necesidad de una Educación para el Consumo. Los consumidores posmodernos hacen su elección en la liquidez y relatividad de sus estilos de vida difusos. Como señalan en su trabajo Ricci, Marinelli y Puliti (2016) aparecen diferentes formas de interpretar al individuo consumidor. Por un lado, consumo como vía de escape, como forma de alcanzar una identidad perdida, por hallar una respuesta, un sentimiento que tiene que ver con el consumo inmediato y fugaz de un producto que calme la sed de satisfacción por una necesidad creada. Por otro lado, dejando al margen esta visión radicalmente pesimista y narcisista del individuo 
consumidor, aparece otra visión, la que presenta un componente ético que ha de ser potenciada y que justifica la necesidad de una educación para el consumo total, la del lado positivo de la liquidez (FABRIS, 2003), representada como el contexto prolífico para el nacimiento de un nuevo consumidor que está libre de restricciones sociales y eventualmente consciente de su elección.

Los contextos ecológico, socioeconómico y cultural están presentes en el alcance del consumo sostenible (GOMBERT-COURVOISIER et al., 2013). Dicho de otra forma, se trabaja y se fomenta un consumo sostenible en estos contextos para avanzar en la resolución/prevención de problemas que, a medio plazo, pueden ser insalvables. A parte de los mencionados contextos se ha de añadir el contexto educativo y comunitario, la escuela y el entorno, el profesorado y las familias, la administración y el tejido socioeducativo no formal. Sin duda, el equitativo consumo de la educación también puede ser llevado a examen, no quedando en una situación muy tranquilizadora. Una amenaza que observamos en la escuela como institución en relación con el consumo es la injerencia del capital y el mercado en la educación, lo cual conlleva la subordinación de la escuela a los intereses del consumo desmedido y mercantilizado, generando la comercialización del conocimiento y la intelectualidad, lo que ha acabado denominándose capitalismo cognitivo (MORGAN, 2016). Este capitalismo cognitivo sitúa en el mismo plano al consumismo, con sus formas productivas y sociales, y a la educación, la cual, cada vez más se ve subyugada por la lógica financiera. Esto trae como desencadenantes una gobernanza y una degradación de lo que a priori serían objetivos educativos y socialmente sostenibles. En lugar de ello, estos objetivos mutan para adaptarse a las lógicas del consumo, implicando peligros para la democracia, la equidad, y la construcción individual y social de la escuela (HEILBRONN, 2016) y fomentando una pedagogía consumista en los contextos escolares que busca inocular en el individuo la necesidad de comprar (SANDLIN, 2005).

Existe la necesidad de educar para el consumo y así lo han indicado también varios organismos internacionales, especialmente aquellos con alcance supranacional (AGUADED, 2011, 2013) como la UNESCO, ONU, o el Parlamento Europeo. A nivel nacional, por ejemplo en España, existen también otras instituciones para la educación del consumidor como son el Instituto Nacional de Consumo, la Red de Educación del Consumidor y los Centros de Formación de Consumo, entre otros. Por todo ello, no podemos seguir considerando el consumo como una actividad frívola sin espacio en la escuela o en otros contextos menos formales, sino que los profesores y educadores deben considerarlo como una necesidad urgente de comprender el mundo de los alumnos y sus relaciones sociales (SANDLIN, 2005; USHER, 2010), y proporcionar las herramientas para el desarrollo de valores y toma de decisiones responsables al respecto (CASTILLEJO et al., 2011).

A pesar de la dificultad de plantear una pedagogía crítica en el aula, el profesorado tiene ante sí la posibilidad de presentar el conocimiento en forma problemática, de tal manera que el alumnado busque una investigación colectiva hacia el cambio social (COLLINSON, 2012; DAMIANIDOU; PHTIAKA, 2016; ROGERS, 2007).

No dejaremos de justificar la necesidad de formar al alumnado como futuros ciudadanos activos, críticos y responsables, con capacidad para adaptarse al devenir social. La educación para el consumo desde una perspectiva crítica es un tema serio que necesita ser estudiado y trabajado por todos los miembros de la sociedad educativa. No en 
vano son muchos los trabajos y esfuerzos de académicos por delimitar y reflexionar sobre estas cuestiones (SANDLIN; MCLAREN, 2010).

La corriente internacional ha hecho que la mayoria de los sistemas educativos hayan adoptado los objetivos de una educación para el crecimiento económico (SPRING, 2013). Sin duda, objetivos nada desdeñables, pero la respuesta crítica debe ser que para fortalecer los andamiajes económicos del Estado no ha de quedar en una situación desdibujada su sistema educativo. El sistema educativo público tiene potencial para, desde posturas críticas con la modalidad de sociedad de consumo actual, convertirse en elemento fundamental para la definición y dinámica de modelos de desarrollo que eleven el nivel y desarrollo económico de un país, pero salvaguardando el establecimiento de niveles de equidad y justicia social. No perdamos de vista que la meta es asegurar una convivencia equilibrada y evitar la creación de tendencias distributivas desfavorables. Una justicia social y una educación para el consumo equitativa, distributiva y compensadora de desigualdades (SIMÓN et al., 2019).

A pesar de que las investigaciones que relacionan la educación con la desigualdad y la clase social son poco esperanzadoras (LYNCH, 2015; MARSH, 2011; RUMBERGER, 2010), pensamos en la importancia de la educación como instrumento para paliar las desigualdades sociales y para mejorar las posibilidades de crecimiento económico y el nivel de vida, lo que permite ampliar las perspectivas de la población y ayudar a alcanzar un entorno más sano, más pleno, y más seguro. El neoliberalismo es peligroso y agresivo desde el punto de vista laboral y educativo, pero no es momento para el pesimismo como indica Apple (2006), sino para construir y defender críticamente la escuela y la educación.

Por tanto, contra esta pedagogía consumista proponemos una pedagogía crítica que potencie la alfabetización cívica, mediática y económica. La primera, como pilar que soporta la democracia y desde donde se pueden establecer las correctas prácticas de consumo. La segunda, la alfabetización mediática (FLORNES, 2016) para capacitar al alumnado con herramientas que le ayuden a gestionar las grandes dosis de información que le sobrevienen hoy día. Esta completará el elenco de soportes para lograr una ciudadanía vital, construida desde los centros educativos. En definitiva, hacer práctica una pedagogía crítica basada en una axiología cimentada en la libertad y dignidad humana, sustentada por la justicia social que permita al sujeto aprender a seleccionar información relevante para después utilizarla como elemento clave de un pensamiento crítico que potencie el consumo razonable.

Finalmente, la alfabetización económica, que desde una pedagogía crítica propone la capacitación para participar activamente en la vida económica y mejorar el bienestar de los individuos. No podemos hacer desaparecer lo que se denomina sociedad de consumo, pero sí podemos convertir, mediante la acción pedagógica que nos ocupa, a esta sociedad en una sociedad de consumo sostenible. Como circunstancia destacable creemos que, en este nivel de alfabetización, así como en los anteriores, el nivel educativo de las familias influye. En la competencia financiera de sus hijos, por ejemplo, a través de los correctos patrones de conducta hacia la evitación del consumismo desenfrenado, la utilidad de los bienes y la coherencia en las actuaciones-ejemplo de los padres o la socialización económica (WEBLEY; NYHUS, 2013). En otro plano, pero continuando con la alfabetización económica, creemos necesario una revisión del concepto de alfabetización económica de 
la OCDE que camina más cerca de los postulados de rendimiento económicos que de los de equidad social, siendo reproductores del pensamiento económico concebido como simples productores desconectados de las dimensiones políticas, sociales y éticas. El resultado son unas políticas premeditadamente clasistas que buscan una clase dominante enriquecida y otra clase suplicante empobrecida.

Desde la acción docente, se debe empoderar a los estudiantes para que sean críticos, reflexivos y socialmente responsables (GONZÁLEZ GÓMEZ et al., 2014). La pedagogía crítica del consumo "[...] ignites the imagination, helps learners to envision and realize new ways of being outside of the commodity"s grip, and encourages learners to become active creators of knowledge rather than passive recipients"3 (SANDLIN et al., 2009, p. 120).

Es necesario introducir en la formación de los futuros docentes y, por tanto, desde la educación superior, el conocimiento y responsabilidad social por saber a qué lugar va nuestro dinero cuando compramos un producto, de dónde ha venido ese producto, cuál es la nacionalidad de la mano de obra del producto, dónde fue manufacturado. Una formación que conciencie e ilustre al docente para convertirlo en un individuo crítico y para que sea capaz de transmitir esa misma capacidad de crítica a sus estudiantes, aunque resulte difícil capacitar profesores para transformar la sociedad en solo unos pocos años de formación inicial (PITTARD, 2015).

El modelo educativo de esta pedagogía del consumo consiste en basar el contexto educativo en un modelo de praxis democrática, con un profesorado como agente socializador crítico y constructivo, un intelectual comprometido con la transformación de la sociedad (GIROUX, 1988), más político y pedagógico enfocado en la empatía para poder emanciparse a sí mismo y a sus alumnos, ampliando su enseñanza al establecimiento del igualitarismo (DAMIANIDOU; PHTIAKA, 2016; GIROUX, 2004). Un profesorado dispuesto a conectar la educación y el consumo más allá de la transmisión de contenidos, compartiendo ideas y ejerciendo poder sobre los principios ideológicos que sustentan las prácticas educativas, capaces de construir una vida más humana (GIROUX; MCLAREN, 1989). La labor del profesor en esta pedagogía crítica del consumo es orientar su actividad educativa hacia un horizonte de la libertad, justicia, tolerancia e igualdad, entre otros, para que el alumnado pueda construir e interiorizar su propio esquema axiológico. Resulta vital formar profesores mentores para transmitir buenas prácticas en relación al consumo de una generación a otra. Enseñar estrategias y formas de provocar una mayor consolidación de pensamiento crítico es fundamental para que el éxito sea alcanzado, cuestión que posee mayor trascendencia si estamos hablando de formar a futuros docentes (GUTIÉRREZ, 2017). Una doble consideración para el estudiante de educación superior que no sólo debe adquirir habilidades para sí (STUPPLE et al., 2017) y que le ayudarán no solo a afrontar mejor las situaciones académicas y personales sino también, formar a los futuros docentes para que estos sean capaces de fomentar el espíritu crítico entre su alumnado.

Desde una pedagogía crítica para el consumo los profesores deben reconocer los peligros del neoliberalismo (SLEETER, 2008), ya que la esfera educativa es donde las

3- "[...] aviva la imaginación, ayuda a los alumnos a visualizar otros puntos de vista que le permitan dejar de ser mercancía manipulable, anima a los alumnos a convertirse en creadores activos de conocimiento en lugar de ser recipientes pasivos" (traducido por los autores). 
fuerzas del neoliberalismo han ido en aumento, y presenta a los profesores como perezosos y necesitados de una disciplina mercantil para ser más eficientes y eficaces (APPLE, 2006).

Frente a la pedagogía consumista, que funciona mejor cuanto más suave se presenta y no se percibe como tal (BLACKER, 2013), el empoderamiento crítico del profesorado puede generar cambios en las dinámicas de poder, habitando el aula y dando instrumentos a los alumnos para su aprendizaje autónomo y crítico, fomentando las capacidades de análisis para mantener su propio carácter crítico y autocrítico.

\section{Últimas reflexiones para concluir}

Educar para el consumo no significa únicamente preparar al alumnado para acceder a los bienes y productos de manera eficaz y eficiente, sino la necesidad de sensibilizarles hacia las implicaciones que tienen sus actos de consumismo. Sensibilizarles de que el actual sistema económico y social no garantiza la supervivencia de próximas generaciones. El consumismo y la globalización están causando un aumento sin precedentes de las diferencias entre ricos y pobres, discriminación económica por motivo de clase social, creencias, raza o sexo (SANDLIN; MAUDLIN, 2012) que favorecen comportamientos, actitudes y prácticas claramente insolidarias.

Coincidimos en la idea que expone Cohen (2006), y es que es necesario que el mundo académico y en este caso el ámbito escolar, no se encuentren solos en la travesía por hallar un consumo responsable. La colaboración de la sociedad civil y del gobierno a través de sus políticas económicas, sociales y concretamente, educativas, es crucial para ello.

La Educación para el Consumo desde una perspectiva crítica ofrece la suficiente solidez para generar una intervención pedagógica fundamentada en un marco de aplicación cognitivo, actitudinal, procedimental y aplicativo. Permitiría generar en el alumnado un desarrollo cognitivo basado en la enseñanza de contenidos sobre el consumo; favorecería un desarrollo actitudinal, ya que plantea el cambio de actitudes y la formación de nuevos hábitos consumistas; un desarrollo procedimental, ya que los discentes ampliarían su habilidad y la tenencia de recursos permitiéndoles tomar decisiones referentes al consumo; y por último, un desarrollo aplicativo poniendo al sujeto en situación de actuar en los contextos que le rodean, aplicando lo aprendido a la vida real. Pasando así de una esfera de aprendizaje centrada en lo meramente informativo y prescriptivo a convertirse en el paradigma de una enseñanza de elevado componente transformador.

Varios autores (GEORGIUS, 2003; SCHUG, 1996) coinciden en que el aprendizaje sistemático de la educación económica, incorporada al currículo y trabajada de manera cotidiana, desarrolla en los estudiantes actitudes positivas hacia el manejo racional de los recursos, así como una mayor capacidad para elegir opciones de comportamiento económico más eficientes. A la hora de introducir en el currículo una educación económica eficiente y adaptada a las demandas de una sociedad equilibrada, se ha de tener presente que este acto no sólo posee ventajas directas sobre el propio saber de lo económico, también se ha advertido una mejora en la comprensión del mundo social, atendiendo a una diferencia trascendental en el modo de ver la vida. Esto potencia la transformación 
del individuo, que pasa de ser un mero observador, a tomar conciencia de sus propias elecciones y de los resultados que estas desencadenan (DENEGRI et al., 2006).

Las sociedades deben seguir promoviendo este consumo responsable y sostenible, pero para ello hay que dotar a la población de mecanismos de defensa, siendo el más potente de todos ellos la educación, y en este caso concreto la Educación para el Consumo.

\section{Referencias}

AGUADED, Ignacio. Media education: an international unstoppable phenomenon UN, Europe and Spain support for edu-communication. Comunicar, Huelva, n. 37, p. 7-8, 2011.

AGUADED, Ignacio. Media programme (UE) - International Support for Media Education. Comunicar, Huelva, n. 40, p. 7-8, 2013.

ALONSO, Luis Enrique; FERNÁNDEZ, Carlos. Consumo e hipermodernidad: una revisión de la teoría de Gilles Lipovetsky. Anuario Filosófico, Navarra, v. 43, n. 2, p. 325-351, 2010.

APPLE, Michael. Understanding and interrupting neoliberalism and neoconservatism in education. Pedagogies, Singapur, v. 1, n. 1, p. 21-26, 2006.

ARRIAZU, Rubén; SOLARI, Mariana. The role of education in times of crisis: a critical analysis of the Europe 2020 strategy. KEDI Journal of Educational Policy, Deoksan-eup, v. 12, n. 2, p. 129-149, 2015.

BADAOUI, Khafid; LEBRUN, Anne-Marie; BOUCHET, Patrick. Clothing style, music, and media influences on adolescents' brand consumption behavior. Psychology \& Marketing, New Jersey, v. 29, n. 8, p. 568-582, 2012.

BAUMAN, Zygmunt. Liquid times: living in an age of uncertainty. Cambridge: Polity Press, 2007.

BAUMAN, Zygmunt. Education in liquid modernity. Review of Education, Pedagogy, and Cultural Studies, Philadelphia, v. 27, n. 4, p. 303-317, 2005.

BEINSTEIN, Jorge. El largo crepúsculo del capitalismo. Buenos Aires: Cartago, 2009.

BERLANGA, Salvador. La educación del consumidor en el aula, en la familia y en la sociedad. Zaragoza: Ministerio de Educación y Mira, 2010.

BERNAL AGUDO, José Luis; LACRUZ, Juan Lorenzo. La privatización de la educación pública: una tendencia en España. Un camino encubierto hacia la desigualdad. Profesorado, Revista de Currículum y Formación del Profesorado, Granada, v. 16, n. 3, p.81-109, 2012.

BESLEY, Tina; PETERS, Michael. The theatre of fast knowledge: performative epistemologies in higher education. Review of Education, Pedagogy, and Cultural Studies, Philadelphia, v. 27, n. 2, p. 111-126, 2005.

BLACKER, David. The falling rate of learning and the neoliberal endgame. Winchester: Zero Books, 2013. 
CALVERT, Sandra. Children as consumers: Advertising and marketing. The Future of Children, New Jersey, v. 18, n. 1, p. 205-234, 2008.

CASTILLEJO, José Luis et al. Educación para el consumo. Educación XXI, Madrid, n. 14, p. 35-58, 2011. COHEN, Daniel. Homo economicus: The (Lost) prophet of modern Times. Cambridge: Polity Press, 2014.

COHEN, Maurie. Sustainable consumption research as democratic expertise. Journal of Consumer Policy, New York, v. 29 n. 1, p. 67-77, 2006.

COLLINSON, Vivienne. Exemplary teachers: teaching for intellectual freedom. Pedagogies, Singapur, v. 7 , n. 2, p. 101-114, 2012.

CRAWFORD, Margaret. The world in a shopping mall. In: SORKIN, Michael (ed.). Variations on a theme park: the new American city and the end of public space. New York: Hill and Wang, 1992. p. 3-30.

DAMIANIDOU, Eleni; PHTIAKA, Helen. A critical pedagogy of empathy: making a better world achievable. Pedagogies, Singapur, v. 11, n. 3, p. 235-248, 2016.

DENEGRI, Marianela et al. Educación económica en la escuela: hacia una propuesta de intervención. Estudios Pedagógicos, Valdivia, v. 22, n. 2, p. 103-120, 2006.

FABRIS, Giampaolo. II nuovo consumatore: verso il postmoderno. Milan: Franco Angeli, 2003.

FEATHERSTONE, Mike. In pursuit of the postmoderm: an introduction. Theory, Culture and Society, New York, v. 5, n. 2-3, p. 195-216, 1988.

FLORNES, K. Promoting civic literacy in teacher education: a framework for personal and professional development. In: INGVALDSEN, Siri; OBERG, Dianne (ed.). Media and information literacy in higher education: educating the educators. Oxford: Chandos; Elsevier, 2016. p. 37-50.

FROMM, Erich. For the love of life. New York: Free Press, 1986.

FROMM, Erich. On disobedience and other essays. New York: Seabury Press, 1981.

GEORGE, Susan. The Lugano Report-New Edition: on preserving capitalism in the twenty-first centu. London: Pluto Press, 2003.

GEORGIUS, George. Pre-college economics instruction and outcome scores. Journal of Business and Economic Studies, Vermont, v. 9, n. 1, p. 65-84, 2003.

GIROUX, Henry. Public pedagogy and the politics of neo-liberalism: making the political more pedagogical. Policy Futures in Education, California, v. 2, n. 3, p. 494-503, 2004.

GIROUX, Henry. Teachers as intellectuals: Toward a critical pedagogy of learning. Granby: Bergin and Garvey, 1988. 
GIROUX, Henry; MCLAREN, Peter. Critical pedagogy, the state, and cultural struggle. Albany: State University of New York Press, 1989.

GIROUX, Henry; POLLOCK, Grace. The mouse that roared: Disney and the end of innocence. Maryland: Rowman and Littlefield Publishers, 2010.

GOMBERT-COURVOISIER, Sandrine et al. Higher education for sustainable consumption: case report on the human ecology master's course (University of Bordeaux, France). Journal of Cleaner Production, Amsterdan, n. 62, p. 82-88, 2013.

GONZÁLEZ GÓMEZ, Yessica et al. La enseñanza de nociones básicas de economía en la formación docente. Una propuesta desde la experiencia de dos instituciones de Educación Superior regional. Profesorado, Revista de Currículum y Formación del Profesorado, Granada, v. 18 n. 3, p. 411-430, 2014.

GÓRNIK-DUROSE, Malgorzata E. Education in possession-how to achieve happiness in the materialistically Oriented world. The New Educational Review, Bydgoszcz, n. 10, p. 91-114, 2006.

GUTIÉRREZ, Patricia. Strategies for teaching and dissemination of artistic heritage by promoting critical and creative thinking among future primary education teachers. Procedia-Social and Behavioral Sciences, Amsterdan, n. 237, p. 717-722, 2017.

HEILBRONN, Ruth. Freedoms and perils: academy schools in England. Journal of Philosophy of Education, New Jersey, v. 50, n. 3, p. 306-318, 2016.

HURSH, David W; HENDERSON, Joseph A. Contesting global neoliberalism and creating alternative futures. Discourse, London, v. 32, n. 2, p. 171-185, 2011.

JONES, Ken et al. Schooling in Western Europe: the new order and its adversaries. London: Palgrave Macmillan, 2008.

LINDSTROM, Martin. Buy ology: truth and lies about why we buy. Broadway: Business, 2010.

LIPOVETSKY, Gilles. Empire de l'éphémère. Princeton: Princeton University Press, 2002.

LYNCH, Kathleen. Control by numbers: new managerialism and ranking in higher education. Critical Studies in Education, London, v. 56, n. 2, p. 190-207, 2015.

MAISURIA, Alpesh. The neo-liberalisation policy agenda and its consequences for education in England: a focus on resistance now and possibilities for the future. Policy Futures in Education, London, v.12, n. 2, p. 286-296, 2014.

MARSH, John. Class dismissed: why we cannot teach or learn our way out of inequality. New York: Monthly Review Press, 2011.

MAUDLIN, Julie; SANDLIN, Jennifer; THALLER, Jonel. Baby culture and the curriculum of consumption: a critical reading of the film Babies. Pedagogy, Culture and Society, London, v. 20, n. 2, p. 211-229, 2012. 
MORGAN, Clara. Testing students under cognitive capitalism: knowledge production of twenty-first century skills. Journal of Education Policy, London, v. 31, n. 6, p. 805-818, 2016.

OLSSEN, Mark; PETERS, Michael A. Neoliberalism, higher education and the knowledge economy: from the free market to knowledge capitalism. Journal of Education policy, London, v. 20, n. 3, p. 313-345, 2005.

PITTARD, Elizabeth. Who does critical pedagogy think you are? Investigating how teachers are produced in critical pedagogy scholarship to inform teacher education. Pedagogies, Singapur, v. 10, n. 4, p. 328-348, 2015.

PORCELLI, Adriana M.; MARTíNEZ, Adriana N. La nueva economía del siglo XXI: análisis de los impactos de la informática en el ambiente: tendencias actuales en tecnologías informáticas verdes, un compromiso con la sustentabilidad. Quaestio luris, Rio de Janeiro, v. 8, n. 4, p. 2174-2208, 2015.

RICCI, Cecilia; MARINELLI, Nicola; PULITI, Lorenzo. The Consumer as Citizen: The Role of Ethics for a Sustainable Consumption. Agriculture and Agricultural Science Procedia, Amsterdan, n. 8, p. 395-401, 2016.

ROGERS, Rebecca. A case study of teaching for literacy acceleration within a critical literacy framework: reconstructing pedagogical approaches. Pedagogies, Singapur, v. 2, n. 4, p. 233-250, 2007.

RUMBERGER, Russell W. Education and the reproduction of economic inequality in the United States: an empirical investigation. Economics of Education Review, Londron, v. 29, n. 2, p. 246-254, 2010.

SALTMAN, Kenneth J. Corporate schooling meets corporate media: standards, testing, and technophilia. Review of Education, Pedagogy, and Cultural Studies, Philadelphia, v. 38, n. 2, p. 105-123, 2016.

SANDLIN, Jennifer. Culture, consumption, and adult education: re-fashioning consumer education for adults as a political site using a cultural studies framework. Adult Education Quarterly, London, v. 55, n. 3, p. $1-17,2005$.

SANDLIN, Jennifer; GARLEN, Julie. Magic everywhere: mapping the Disney curriculum. Review of Education, Pedagogy, and Cultural Studies, Philadelphia, v. 39, n. 2, p. 190-219, 2017.

SANDLIN, Jennifer; MAUDLIN, Julie. Consuming pedagogies: controlling images of women as consumers in popular culture. Journal of Consumer Culture, London, v. 12, n. 2, p. 175-194, 2012.

SANDLIN, Jennifer; MCLAREN, Peter (ed.). Critical pedagogies of consumption: living and learning in the shadow of the" shopocalypse". New York: Routledge, 2010.

SANDLIN, Jennifer et al. To find the cost of freedom: theorizing and practicing a critical pedagogy of consumption. Journal for Critical Education Policy Studies, London, v. 7, n. 2, p. 98-125, 2009.

SANTA CRUZ, Eduardo; OLMEDO, Antonio. Neoliberalismo y creación del 'sentido común': crisis educativa y medios de comunicación en Chile. Profesorado, Granada, v. 16, n. 3, p. 145-168, 2012. 
SCHOR, Juliet. Born to buy: The commercialized child and the new consumer culture. New York: Scribner, 2005.

SCHUG, Mark. Introducing children to economic reasoning: some beginning lesson. The Social Studies, London, v. 87, n. 3, p. 114-118, 1996.

SIMÓN, Cecilia et al. Equidad, educación inclusiva y educación para la justicia social. ¿Llevan todos los caminos a la misma meta? Revista Internacional de Educación para la Justicia Social, Madrid, v. 8, n. 2, p. 17-32, 2019.

SLEETER, Christine. Equity, democracy, and neoliberal assaults on teacher education. Teaching and Teacher Education, London, v. 24, n. 8, p. 1947-1957, 2008.

SPRING, Joel. Corporatism, social control, and cultural domination in education: from the radical right to globalization: the selected works of Joel Spring. London: Routledge, 2013.

SPRING, Joel. Educating the consumer-citizen: a history of the marriage of schools, advertising, and media. London: Routledge, 2003.

STUPPLE, Edwuard J. et al. Development of the Critical Thinking Toolkit (CriTT): a measure of student attitudes and beliefs about critical thinking. Thinking Skills and Creativity, Amsterdan, n. 23, p. 91-100, 2017.

TOFFLER, Alvin. Future shock. New York: Random House, 1970.

USHER, Robin. Consuming learning. In: SANDLIN, Jennifer; MCLAREN, Peter. (ed.). Critical pedagogies of consumption: living and learning in the shadow of the 'shopocalypse'. New York: Routledge, 2010. p. 36-46.

VIÑAO, Antonio. El concepto neoliberal de calidad de la enseñanza: su aplicación en España (1996-1999). Témpora, Santa Cruz de Tenerife, n. 4, p. 63-88, 2001.

VIÑAO, Antonio. El desmantelamiento del derecho a la educación: discursos y estrategias neoconservadoras. AREAS, Murcia, n. 31, p. 97-107, 2012.

WAINES, Nahid. Development of economic concepts among Egyptian children. Journal of Cross Cultural Psychology, New York, v. 15, n. 1, p. 47-64, 1984.

WEBLEY, Paul; NYHUS, Ellen K. Economic socialization, saving and assets in European young adults. Economics of Education Review, London, n. 33, p. 19-30, 2013.

WRIGHT, Robin R.; SANDLIN, Jennifer. (Critical) learning in/through everyday life in a global consumer culture. International Journal of Lifelong Education, London, v. 36, n. 1-2, p. 77-94, 2017. 
Recibido en: 25.03.2020

Revisado en: 12.05 .2020

Aprobado en: 21.07.2020

Miguel Martín-Sánchez es profesor titular en el área de Teoría e Historia de la Educación, en el Departamento de Ciencias de la Educación de la Universidad de Extremadura. Es coordinador del grupo de investigación GEXTHE (Grupo Extremeño de investigación en Teoría e Historia de la Educación).

Laura Casares-Ávila es doctora en Educación por la Universidad de Extremadura, máster Universitario de Investigación en Ciencias Sociales y Jurídicas por la Universidad de Extremadura y licenciada en Psicología por la Universidad de Salamanca.

Jorge Cáceres Muñoz es doctor en Educación por la Universidad de Extremadura y profesor del área de Teoría e Historia de la Educación en la Facultad de Formación del Profesorado de la Universidad de Extremadura. 